\title{
3D Confocal Raman Tomography to Probe Field Enhancements inside Supercluster Metamaterials
}

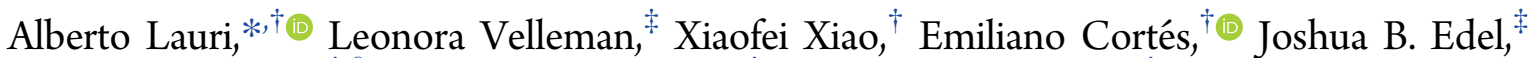 \\ Vincenzo Giannini, ${ }^{\dagger, \S}$ Aliaksandra Rakovich, ${ }^{\dagger \odot}$ and Stefan A. Maier \\ ${ }^{\dagger}$ Department of Physics, Imperial College London, London SW7 2AZ, U.K. \\ ${ }^{\ddagger}$ Department of Chemistry, Imperial College London, South Kensington Campus, London SW7 2AZ, United Kingdom \\ ${ }^{\S}$ Instituto de Estructura de la Materia (IEM-CSIC), Consejo Superior de Investigaciones Científicas, Serrano 121, 28006 Madrid, \\ Spain
}

\section{Supporting Information}

ABSTRACT: Spherical colloidal superclusters, composed from sub-100 nm plasmonic nanoparticles, have been proposed to possess collective plasmonic modes imbued with large field enhancements and tunable spectral response extending from the visible to infrared regions. Here, we report the experimental verification of collective near-IR plasmonic modes inside single superclusters, with dimensions ranging from $0.77 \mu \mathrm{m}$ up to $2 \mu \mathrm{m}$. Raman reporters, coated onto the nanoparticle building blocks, were used as local probes of the electric field enhancement inside
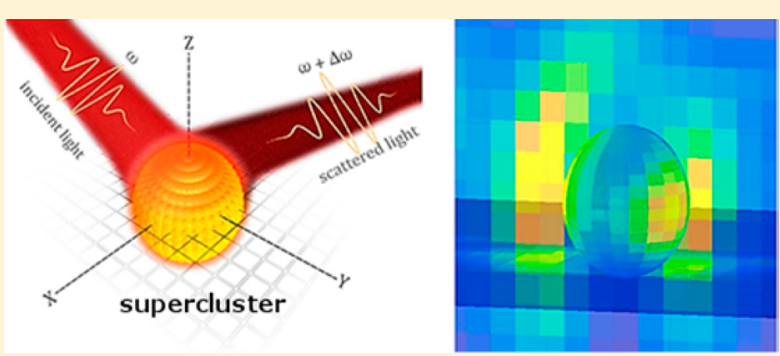
the metamaterial. By performing diffraction-limited 3D Raman tomography we were able to build up the electric field intensity distribution within the superclusters. We demonstrate that plasmonic responses of superclusters vary according to their size and excitation wavelength, in accordance with theoretical predictions of their tunable optical properties. The existence of three-dimensional internal collective modes in these superclusters enables the excitation of a large number of electromagnetic hot-spots, validating these self-assembled structures as promising candidates for molecular spectroscopy.

KEYWORDS: self-assembly, nanoparticles, SERS, collective plasmon modes

S elf-assembly of nanoscale building blocks into hierarchical superstructures is quickly becoming one of the most pursued topics in nanomaterials science. ${ }^{1-3}$ The possibilities obtainable when individual components arrange themselves into an ordered assembly are limitless and of great interest, as has been demonstrated with biological self-assembled structures such as DNA, opals, and proteins. ${ }^{4-6}$ An extensive amount of literature exists on different strategies for the synthesis of selfassembled structures; ${ }^{7,8}$ however, complications arise when a high degree of control is sought over the process. For example, controlled 3D self-assembly of nanoparticles (NPs) is possible utilizing templates with specificity to certain types of particles, such as those achieved by DNA-origami techniques, among others. ${ }^{9-12}$ However, advanced techniques such as these restrict the surface functionalization of the NPs, thereby negating one of the most important benefits that these structures offer: the ability to control the functionality of the building blocks. ${ }^{13}$ Moreover, such particle-by-particle self-assembly methods limit the amount of constituents forming the superstructures to only a few of them, i.e., in the range of 10's. Recently, the generation of spherical colloidal crystals with functionalizable building blocks has been demonstrated using a novel self-assembly process based on emulsion formation in the nanoparticle solution. ${ }^{13}$ The introduction of plasmonic materials as building blocks of a spherical colloidal cluster is easily achievable, facilitating the engineering of materials with complex optical properties and revealing exciting new directions in sensing and metamaterials research. ${ }^{14-16}$

Spherical colloidal clusters, built from plasmonic building blocks, have been proposed to possess remarkable collective supermodes with large local field enhancements ${ }^{13,17-19}$ and a spectral response extending from the near-infrared to deep into the mid-infrared region. ${ }^{11}$ As such, these superstructures hold great promise as a sensing platform that is capable of addressing the whole spectral domain of vibrational molecular fingerprints, ${ }^{20,21}$ while simultaneously exploiting the advantages of their plasmonic constituents. Previously, dark-field spectra of plasmonic superclusters ${ }^{13}$ indicated the existence of collective plasmonic modes in these structures; however, no direct experimental verification has been obtained to date. This ability to access thousands of hot-spots upon the excitation of a collective mode inside the supercluster enables high signal-tonoise ratios, something that is of great significance in sensing applications.

Received: May 20, 2017

Published: July 6, 2017 
a)

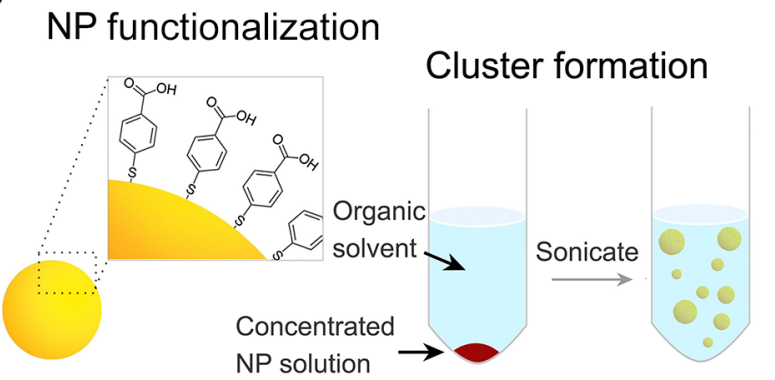

b)

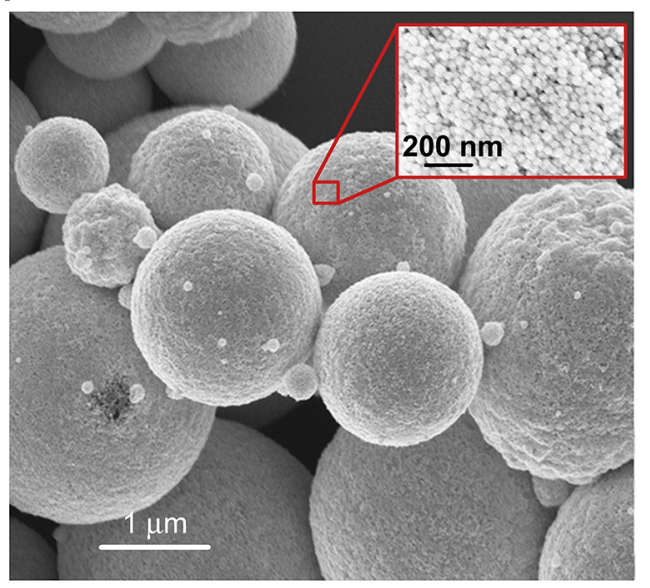

c)

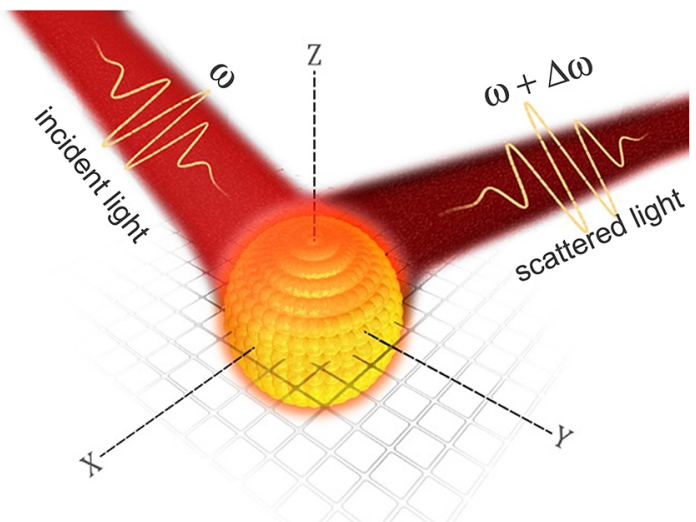

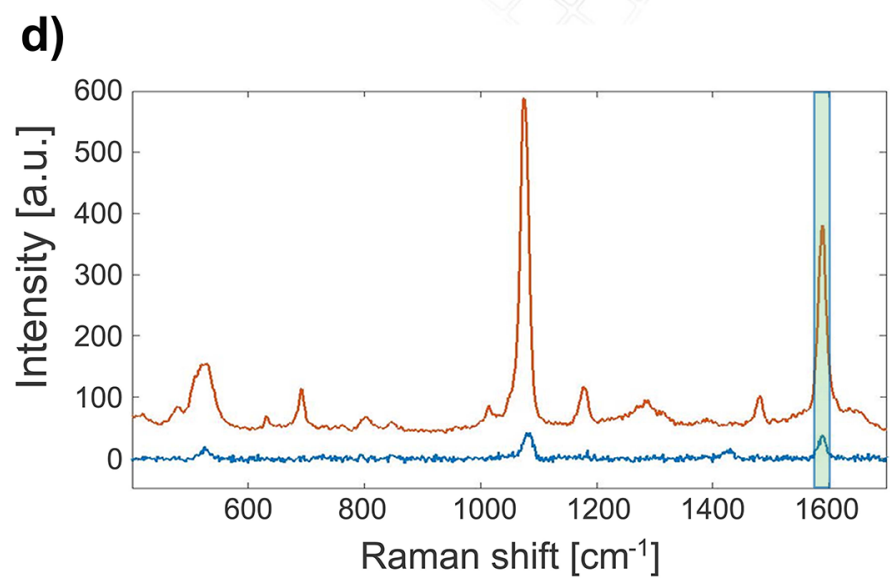

Figure 1. Fabrication and characterization of spherical metallic superclusters. (a) Schematic representation of the functionalization and self-assembly of metallic nanoparticles into superclusters (see Methods section for methodology details). (b) SEM image of the superclusters and typical size dispersion observable after drop-casting. The inset shows a zoomed-in area of one supercluster, with the small gold NPs tightly packed and separated by a distance dictated by the size of the ligands on the NPs' surfaces. (c) Schematic representation of 3D mapping of the plasmonic mode using the SERS signal from the 4-MBA Raman marker. The spherical supercluster is excited from the top at different wavelengths, and the signal from a specific XY plane is recorded using a confocal microscope setup. A series of equally spaced $2 \mathrm{D} \mathrm{XY}$ planes in the $\mathrm{Z}$ direction is used to completely map the plasmonic mode of the cluster. (d) SERS signal for the 4-MBA Raman marker measured on semi-isolated $40 \mathrm{~nm}$ gold nanoparticles (blue line) and on $40 \mathrm{~nm}$ gold particles assembled into a spherical supercluster of $770 \mathrm{~nm}$ diameter (red line). The signal of the 4-MBA Raman marker is characterized by peaks at $1100 \mathrm{~cm}^{-1}(\mathrm{~A})$ and at $1590 \mathrm{~cm}^{-1}(\mathrm{~B})$.

Different techniques have been employed in the past to visualize the near fields of simple plasmonic structures; ${ }^{2-24}$ however, obtaining similar field maps of complex threedimensional (3D) plasmonic materials ${ }^{25-27}$ still remains very challenging. One possibility that has been exploited in the past is photoluminescence microscopy, ${ }^{28,29}$ which relies on detecting the fluorescence of dye molecules next to plasmonic structures. However, in the case of colloidal plasmonic superclusters, the compactness of the array of nanoparticles would result in a strongly quenched fluorescence of the dye molecules. ${ }^{13,30,31}$ Other techniques utilized for this purpose are electron energy loss spectroscopy (EELS), ${ }^{32}$ cathodoluminescence, ${ }^{33}$ and near-field scanning optical microscopy (SNOM). ${ }^{34-36}$ However, these methods are limited to the surface of the plasmonic structures, making it difficult to inspect collective modes inside the superclusters. In contrast, techniques relying on surface-enhanced scattering, such as SERS (surface-enhanced Raman spectroscopy) ${ }^{37-39}$ and surface-enhanced infrared spectroscopy, ${ }^{40,41}$ are perfectly suited to $3 \mathrm{D}$ inspection of electric field distribution inside complex structures. Indeed, SERS signals give a direct and extremely sensitive measure of the near-field intensity enhancement, ${ }^{42-45}$ due to fourth power dependency on the (local) electric field.
Furthermore, Raman scattering is an optical property that is not afflicted by quenching issues and, thus, is easily adaptable to 3D inspection of complex structures.

Here we present an approach to performing $3 \mathrm{D}$ tomography of near-field intensity inside a supercluster by exploiting the SERS signal of a molecular probe (4-mercaptobenzoic acid, 4MBA). Specifically, we extract plane-by-plane intensity maps of the local plasmonic near field by measuring the Raman spectra of our probe molecule with a confocal microscope. In this way, we successfully map collective modes inside supercluster structures of various dimensions, providing experimental proof of their existence.

\section{RESULTS AND DISCUSSION}

The spherical colloidal superclusters were fabricated by selfassembly following the procedure outlined by Turek et al. ${ }^{13}$ Our only deviation from this fabrication procedure was the prefunctionalization of $40 \mathrm{~nm}$ gold nanoparticles with the 4MBA molecular probe prior to the assembly process (Figure 1a), as outlined in the Methods section. 4-MBA was chosen, as it is one of the most widely used Raman probes for evaluating SERS in plasmonic structures over a large range of wavelengths. ${ }^{46}$ Its popularity is based on an easy and uniform self- 


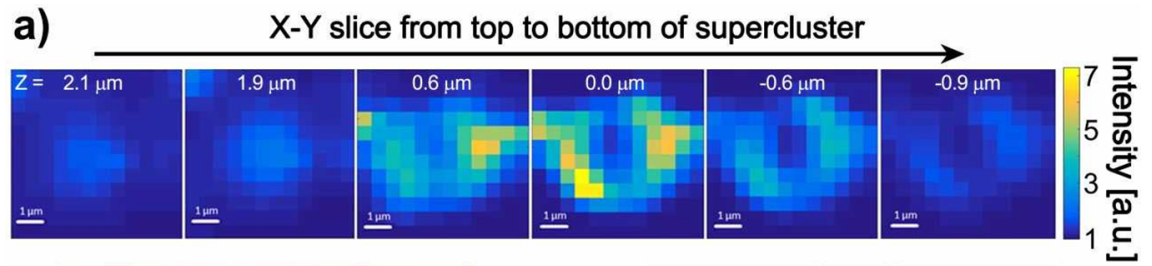

b)

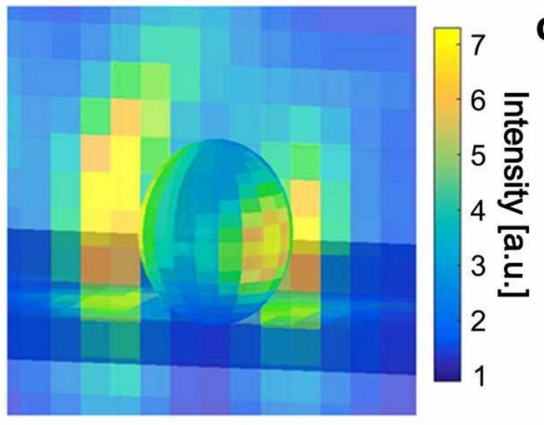

c)

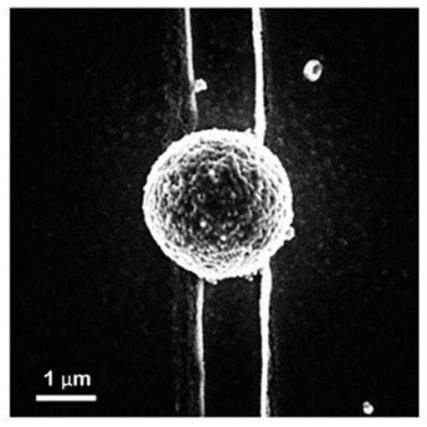

Figure 2. Experimental Raman tomography mapping of a $1.6 \mu \mathrm{m}$ diameter supercluster. (a) XY Raman maps following the intensity of the B peak of the 4-MBA molecular probe, when excited with a $785 \mathrm{~nm} \mathrm{CW}$ laser. The $2 \mathrm{D}$ planes are taken at different $\mathrm{Z}$ values, with $\mathrm{Z}$ being the axis along the height of the spherical cluster and $\mathrm{Z}=0$ being slightly lower than the expected center of the supercluster. (b) $3 \mathrm{D}$ reconstruction of the plasmonic mode excited in the spherical supercluster by staking all the 2D Raman map along the $\mathrm{Z}$ axis. (c) Scanning electron microscope image of the spherical supercluster $(1.61 \mu \mathrm{m}$ diameter $)$.
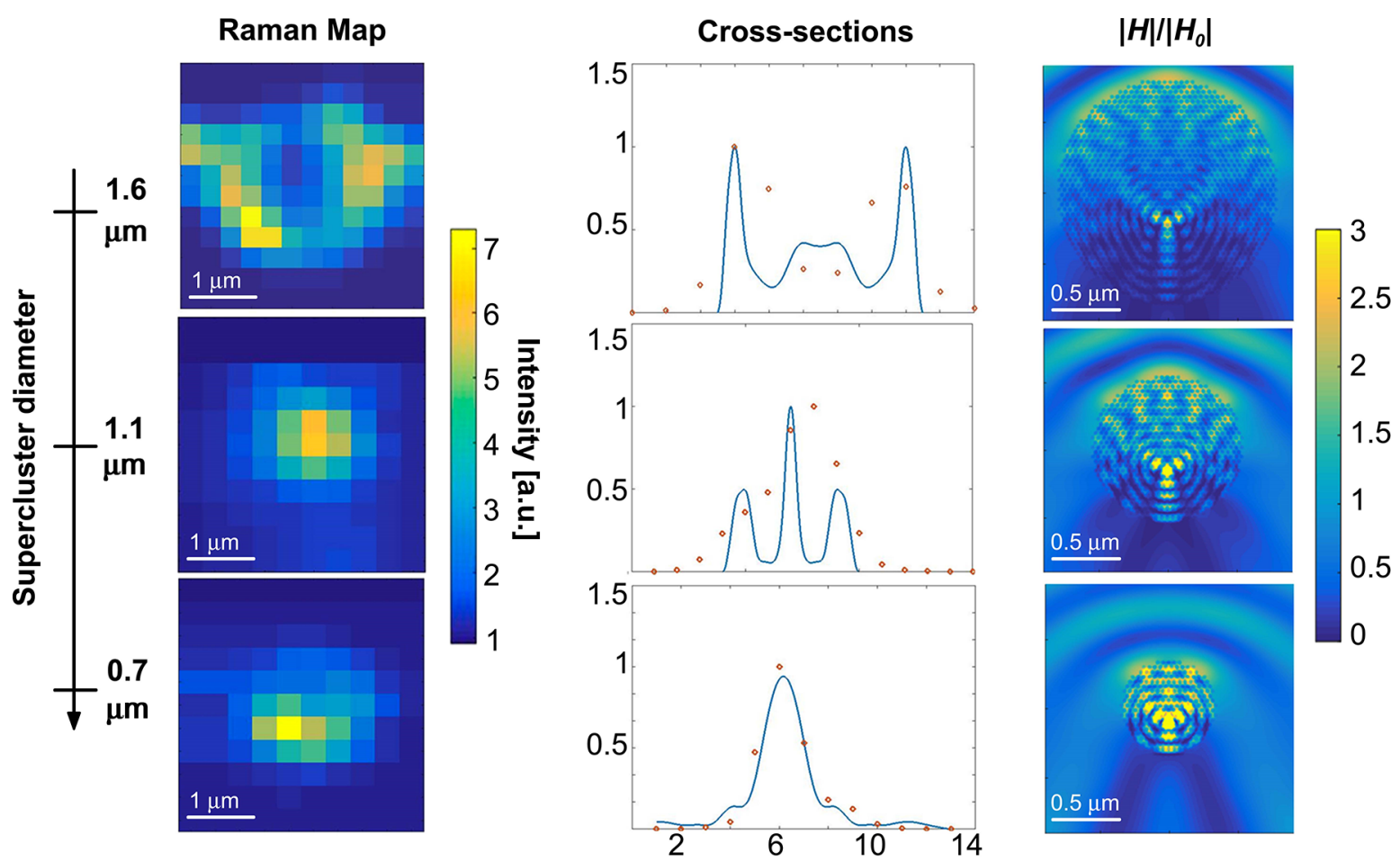

Figure 3. Generation of a mode inside a spherical supercluster as the size of the cluster is reduced from $1.6 \mu \mathrm{m}$ to $0.77 \mu \mathrm{m}$. In the first column, experimental XY Raman maps are presented for different supercluster sizes $(1.61,1.11$, and $0.77 \mu \mathrm{m})$. Raman maps were obtained by following the intensity of the B peak of 4-MBA when excited with a $785 \mathrm{~nm} \mathrm{CW}$ laser and through the center of each cluster. The last column shows the corresponding XZ maps obtained from FDTD simulations of the magnetic field enhancements. The middle column compares the experimental (scatter points) and theoretical (solid lines) cross sections of the electric field enhancement maps through the center and along the $\mathrm{X}$ direction of the clusters.

assembly on $\mathrm{Ag}$ and $\mathrm{Au}$ surfaces (due to its thiol group), ensuring the presence of the Raman probe inside the extremely small gaps of superclusters. Furthermore, it has a high Raman cross-section in the IR and mid-IR, ${ }^{47,48}$ i.e., in the spectral regions where the superclusters are expected to have optical resonances.
The presence of 4-MBA on the nanoparticle surface was confirmed by measuring Raman scattering from a layer of nanoparticles drop-casted on a glass substrate. The blue curve in Figure 1d shows a Raman spectrum typical for 4-MBA, characterized by two strong distinctive peaks at $1100 \mathrm{~cm}^{-1}$ (peak A) and at $1590 \mathrm{~cm}^{-1}$ (peak B), corresponding to the $v_{12}$ and $v_{8 \mathrm{a}}$ aromatic ring vibrations, respectively. ${ }^{49-51}$ 

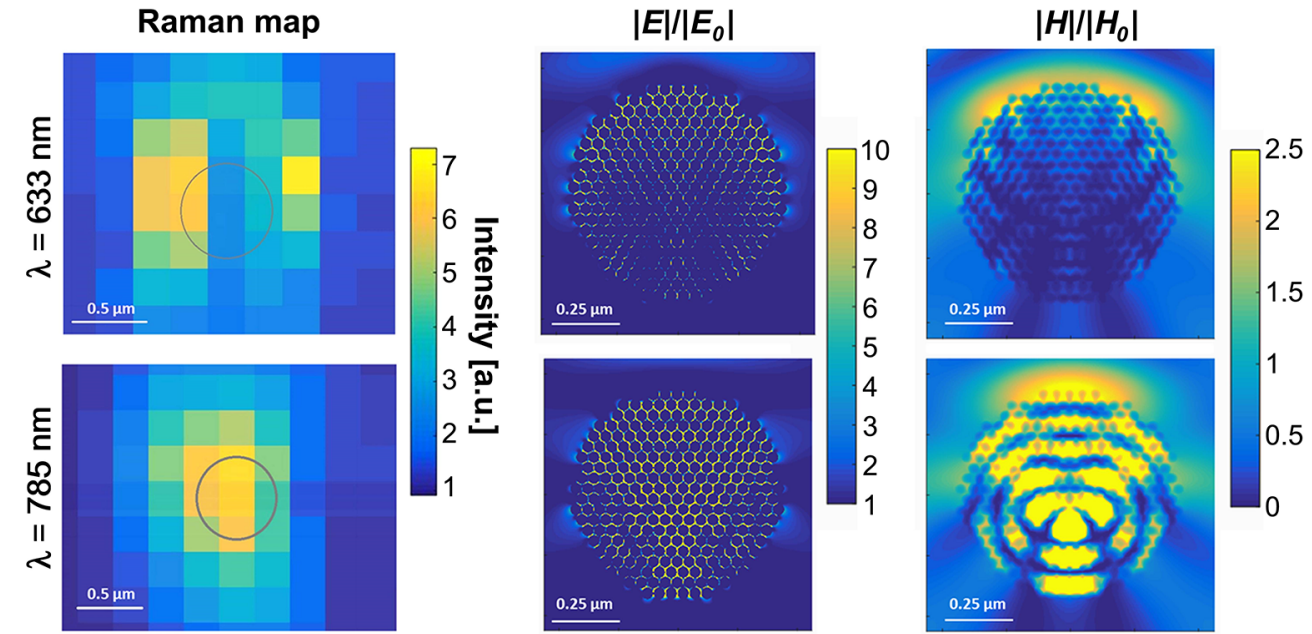

Figure 4. Internalization of modes in a $0.77 \mu \mathrm{m}$ supercluster with increasing excitation wavelength. Top and bottom rows correspond to 633 and 786 $\mathrm{nm}$ excitation, respectively. The first column shows the experimentally obtained Raman maps in the XZ plane. The other two columns compare these results to FDTD simulations: the calculated electric field enhancements $\left(E / E_{0}\right)$ and magnetic field enhancements $\left(H / H_{0}\right)$ are shown in the second and third columns, respectively.

Next, the prefunctionalized building blocks were selfassembled into spherical colloidal clusters using the previously mentioned methods. A large drop containing clusters with sizes between 0.5 and $3 \mu \mathrm{m}$ was then drop-casted onto a glass slide, patterned with a grid to aid their localization throughout the characterization process (see Methods for further details). The red curve in Figure 1d shows a representative SERS spectra from an assembled superstructure, exhibiting no significant changes in spectral features with respect to the nonassembled particles. We should note that, although the SERS intensity in the case of the supercluster is higher, no direct comparison of the enhancement values can be made, as the number of gold nanoparticles in our focal volumes for the two cases is different (due to the three-dimensionality of the cluster).

Plasmonic near-fields of single superclusters were mapped by scanning the $\mathrm{X}-\mathrm{Y}$ plane and then repeating these measurements at different $\mathrm{Z}$-positions across the height of the cluster. The resolution in the $\mathrm{X}, \mathrm{Y}$, and $\mathrm{Z}$ direction for our measurement was diffraction limited as for an optimized confocal setup ( $280 \mathrm{~nm}$ for $633 \mathrm{~nm}$ excitation wavelength and $340 \mathrm{~nm}$ for $785 \mathrm{~nm}$ excitation wavelength).

2D Raman intensity maps were then extracted from the scattering spectra by integrating the SERS signal across peak $B$ of 4-MBA, using a digital filter extending from 1561 to 1621 $\mathrm{cm}^{-1}$ (as shown in green in Figure 1d). Figure 2a shows some of the resulting maps obtained for a $1.6 \mu \mathrm{m}$ supercluster at 785 $\mathrm{nm}$ excitation. These series of $2 \mathrm{D}$ maps were then joined using Matlab to create a 3D tomographic image of the enhanced SERS signal (Figure $2 \mathrm{~b})$ for this cluster. The diameter $(1.6 \mu \mathrm{m})$ and, more importantly, the position of the cluster were confirmed by Scanning electron microscopy (SEM) (Figure 2c), with the latter being used to correct for any drifts of the scanner during data acquisition. As the Raman process for a molecule next to a plasmonic structure benefits from both the emission and excitation enhancements, ${ }^{52}$ the SERS intensity can be approximated to be $|E|_{\text {Exc }}^{2}|E|_{\text {Em }}^{2} \approx|E|_{\text {SERS }}^{4}$ (see Supplementary Figure 6 for further discussion of this approximation). This fourth power dependency outlines that the intensity of SERS (i.e., a molecule in a plasmonic environment) is a very sensitive parameter of the strength of the local field. Given this relation between SERS signal and plasmonic enhancements, ${ }^{38,53-55}$ the $3 \mathrm{D}$ tomography is a reliable representation of the plasmonic mode excited in the spherical cluster.

Figure 2a,b outline the enhancement of the Raman signal in the $1.6 \mu \mathrm{m}$ supercluster at $785 \mathrm{~nm}$ excitation. The strongest signal is present on the edges and on top of the cluster, while only a weak signal is present inside. This suggests that, at 785 $\mathrm{nm}$, a cluster of this dimension is mainly scattering light and not absorbing it, resulting in a shallow excitation of the electric field inside the structure and an enhanced signal present only on the surface.

In order to investigate the preliminary results obtained by SERS mapping, finite-difference time domain (FDTD) simulations where performed, ${ }^{56}$ allowing us to assess both the spectral and near-field properties of the structures. The numerical simulations substantiated the experimental results obtained in Figure $2 b$, where no collective mode was observed in the infrared for a supercluster with a diameter of $1.6 \mu \mathrm{m}$ (first row, third column Figure 3).

Following this finding, we expanded the theoretical studies in order to understand when the excitation of a collective mode inside the supercluster was possible. In Supplementary Figure $2 \mathrm{a}$ we show the normalized absorption cross section when the diameter of the cluster is varied between 1.6 and $0.7 \mu \mathrm{m}$. We found that, in the mid-IR, the absorption of the cluster varies strongly with the diameter and coherent collective modes appear at $785 \mathrm{~nm}$ only when the diameter of the cluster is less than $1 \mu \mathrm{m}$. This suggests that, as the diameter of the supercluster is reduced, the excited collective mode starts to internalize, and, as the size is reduced further, this process becomes more efficient. This stipulation was supported by the results of FDTD simulations of the near-field intensities inside superclusters of varying diameters $(1.6,1.1$, and $0.7 \mu \mathrm{m})$, excited at $785 \mathrm{~nm}$ (see last column of Figure 3 and Supplementary Figure 4). It should be noted that the simulations of the near-field intensities hold also when the structure simulated is not perfectly crystalline (Supplementary Figures 8 and 9).

To confirm these theoretical expectations, we perform Raman tomography on similarly sized superclusters. The first column of Figure 3 presents large-area 2D Raman scans of the 
three different superclusters excited at $785 \mathrm{~nm}$, taken exactly through the vertical center of each (SEM images of the three clusters can be found in Supplementary Figure 3). From the SERS map we can clearly observe that as the diameter of the cluster is reduced, an internal collective mode starts to be excited. This can be seen more clearly in the second column of Figure 3, where the integrated Raman intensity of the B peak of 4-MBA is plotted across the supercluster center. As the cluster dimension reaches $1.1 \mu \mathrm{m}$ in diameter, an internal collective mode is excited, and all the pixels across the center of the particle show a coherent field enhancement. Moreover, as the size of the supercluster is reduced even further (to $0.77 \mu \mathrm{m}$ ), the field enhancement from the central pixel of the spherical cluster likewise increases further. Such behavior is consistent with the simulations presented in the last column of the same figure, where similar observations can be made about the magnetic field distribution inside the different clusters.

Finally, the near-field distribution for the smallest spherical cluster was evaluated $(0.77 \mu \mathrm{m}, \mathrm{SEM}$ image of this cluster can be found in Supplementary Figure 1a) for different excitation wavelengths. In particular, in Figure 4, we compare the mapping by SERS and the FDTD simulations when the cluster with a diameter of $0.77 \mu \mathrm{m}$ is excited at $633 \mathrm{~nm}$ and $785 \mathrm{~nm}$. The first column of Figure 4 presents the two SERS maps of the plasmonic mode visualized on the $\mathrm{X}-\mathrm{Z}$ plane at the center of the cluster, as extrapolated by the $3 \mathrm{D}$ reconstruction. The dark gray circle represents the expected position of the particle with respect to the grid structure around it, as determined from SEM microscopy images (Supplementary Figure 1a).

From the SERS maps it can be seen that when the spherical cluster is excited at $633 \mathrm{~nm}$, the electric field enhancement is present only on the edges of the particles with no seepage inside and a signal enhancement is spatially defined only by our laser penetration depth (similar to what is observed in a supercrystal system with normal SERS measurements ${ }^{57}$ ). This is corroborated by the electric field FDTD simulations presented in the second column of Figure 4. Indeed, from the calculations, it can be seen that the field is scattered from the spherical cluster toward the excitation direction, and electric field enhancement is present only on the outer layer of particles forming the cluster. On the other hand, when the excitation wavelength is switched to $785 \mathrm{~nm}$, a completely different profile is obtained. From the Raman maps we observe the presence of field enhancement over the entirety of the cluster with a stronger field localized at the center; in other terms, the collective plasmonic mode is excited inside the cluster. The presence of an internal collective mode can also be seen from the field enhancement simulations, where for $785 \mathrm{~nm}$ we observed strong field enhancements in almost all the inside gaps of the spherical cluster.

This observation can become even clearer by looking at the magnetic field distribution whose variations are less abrupt. In the third column of Figure 4 we show the magnetic field distributions inside the $0.77 \mu \mathrm{m}$ cluster for the two excitation wavelengths. While at $633 \mathrm{~nm}$ the magnetic field distribution clearly shows that no plasmonic collective mode is excited inside the supercluster, at $785 \mathrm{~nm}$ we start to see the field distribution of a mode similar to what can be found in a transparent cylinder ${ }^{13,56}$ and the beginning of an internal collective mode. Indeed, if not for the presence of a collective mode, the internal excitation of the structure would not be possible, as the localized surface plasmon resonance of the single nanoparticles is centered at $530 \mathrm{~nm}$ with a negligible extinction contribution at $785 \mathrm{~nm}$. This internalization of a mode was found to be consistent for clusters of comparable size, despite small variations in cluster geometry due to different sizes and packing imperfections (see Supplementary Figures 5 and 8 ).

These optical modes can be extremely useful, as the field enhancements are localized in each gap between the NPs. This is relevant to spectroscopy because large hot-spot densities lead to larger interactions with the field and therefore improved detection. ${ }^{58}$ Furthermore, the porous structure of the supercluster outlines its ability to take up and host a huge number of molecules and exploits this large hot-spot density. ${ }^{59}$ To prove superclusters possess these characteristics, we performed $\mathrm{pH}$ sensing measurements, taking advantage of the previously characterized collective mode at $785 \mathrm{~nm}$.

The $\mathrm{pH}$ sensitivity in our measurement comes from the changes in the Raman spectrum of 4-MBA due to protonation/ deprotonation $^{60}$ of its carboxylic acid end group. Indeed, as previously reported, ${ }^{61}$ when $4-\mathrm{MBA}$ is deprotonated (i.e., when $\left.\mathrm{pH}>\mathrm{p} K_{\mathrm{a}}\right)$, a new vibrational mode appears at $1430 \mathrm{~cm}^{-1}$, representing the stretching $\left(v_{8 \mathrm{a}}\right)$ of a deprotonated group (COO-). This can be clearly observed in Figure 5a, where

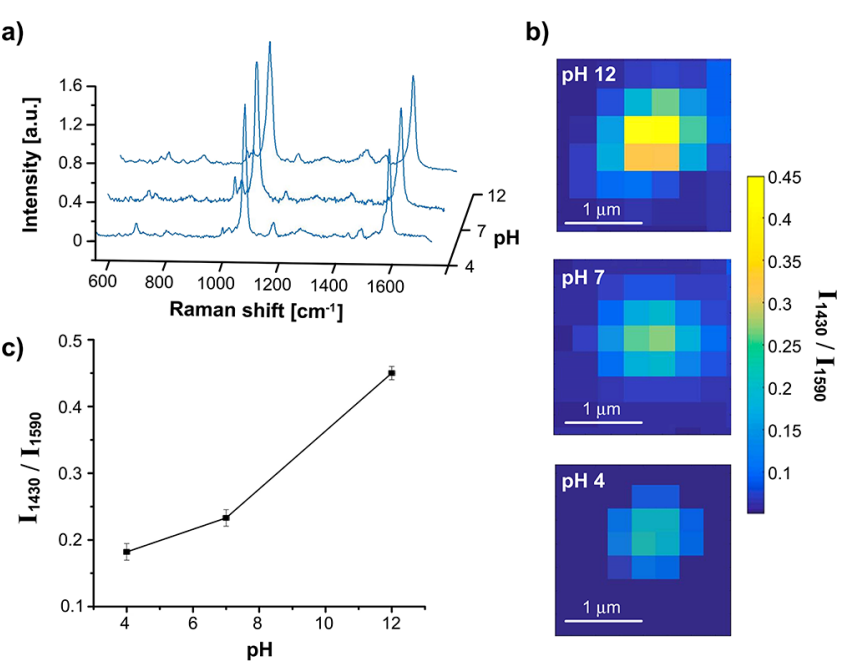

Figure 5. $\mathrm{pH}$ sensing with a single $0.75 \mu \mathrm{m}$ diameter supercluster excited at $785 \mathrm{~nm}$. (a) Raman spectra for clusters exposed to solutions of three different $\mathrm{pH}$ values. As the $\mathrm{pH}$ was increased, a peak at 1430 $\mathrm{cm}^{-1}$ appeared, corresponding to the stretching mode of MBA after deprotonation of its carboxyl group. (b) 2D Raman maps on the same supercluster for the different $\mathrm{pH}$ values show that the signal from the stretching mode is originating from the entirety of the supercluster. (c) Increase of the intensity of the $\mathrm{pH}$-sensitive stretching mode of 4-MBA relative to the $\mathrm{pH}$-insensitive mode at $1590 \mathrm{~cm}^{-1}$, when exposing the supercluster to progressively higher $\mathrm{pH}$ solutions.

three Raman spectra are presented for the center of the same cluster that has been exposed to three different $\mathrm{pH}$ solutions: as the $\mathrm{pH}$ value is increased, the appearance of the new vibrational mode at $1430 \mathrm{~cm}^{-1}$ becomes clearer.

In order to confirm that the Raman signal from the new vibrational mode originated from the entirety of the supercluster structure, we also obtained 2D Raman maps (Figure 5b) for clusters exposed to different $\mathrm{pH}$ values. Tracking the $v_{8 \mathrm{a}}$ (COO-) mode progressively confirmed the diffusion of the protons inside the clusters, since the signal was coming from the very center of the supercluster and its distribution was consistent with the previously reported mode at $785 \mathrm{~nm}$. This 
experiment, as well as the observation of coherent collective modes inside a cluster functionalized with 4-MBA after the process of self-assembly (Supplementary Figure 5), proves the ability of this system to host small molecules in its porous structure.

Finally, we quantitatively evaluated the response of the supercluster to $\mathrm{pH}$ variations by measuring the ratio of intensities of the $\mathrm{pH}$-dependent $v_{8 \mathrm{a}}(\mathrm{COO}-)$ peak at 1430 $\mathrm{cm}^{-1}$ and the internal standard vibrational mode at $1590 \mathrm{~cm}^{-1}$. Figure $5 \mathrm{c}$ shows that, unlike the previously reported measurements involving single gold nanoparticles, ${ }^{60}$ the deviation of our $\mathrm{pH}$ sensing measurements was statistically insignificant compared to the change in the qualitative response of clusters. Thus, superclusters are capable of increasing the $\mathrm{pH}$ sensitivity of the constituent gold nanoparticles by presenting a coherent ensemble of hot-spots, thus representing a valuable resource for high-sensitivity SERS measurements.

\section{CONCLUSIONS}

In summary, this study builds on our understanding of the selfassembled colloidal structures, by providing the first direct experimental proof for the existence of their collective supermodes in the visible and near IR. It also establishes SERS tomography as an invaluable technique for mapping out plasmonic modes of three-dimensional structures characterized by extremely small gaps. Moreover, as SERS allows sensitive detection of molecules, the remarkable and tunable modes excitable inside the spherical colloidal clusters provide an efficient platform for ultrasensitive molecular spectroscopy. To this end, we presented proof-of-principle implementation of the superclusters as an efficient platform for $\mathrm{pH}$ sensing of the surrounding medium.

\section{METHODS}

Nanoparticle Formation. The $42 \mathrm{~nm}$ gold nanoparticles were synthesized via a seed-mediated growth approach based on the work of Brown et al. ${ }^{62}$ First, $16 \mathrm{~nm}$ seed gold nanoparticles were synthesized by the Turkevich-Frens method. ${ }^{63,64}$ Briefly, a $500 \mathrm{~mL}$ solution of $0.1 \mathrm{w} / \mathrm{v} \%$ gold chloride was brought to reflux under stirring; then $10 \mathrm{~mL}$ of 1 $\mathrm{w} / \mathrm{v} \%$ sodium citrate was added. The reaction was allowed to proceed over $30 \mathrm{~min}$, cooled to room temperature, and filtered through a $0.2 \mu \mathrm{m}$ mixed cellulose ester membrane (Advantec). For seeded growth of the $42 \mathrm{~nm}$ gold nanoparticles, $60 \mathrm{~mL}$ of seed was added to $540 \mathrm{~mL}$ of water. Under vigorous stirring, 6 $\mathrm{mL}$ of $1 \mathrm{M}$ hydroxylamine hydrochloride solution was added. The solution was allowed to mix for $1 \mathrm{~min}$; then $5 \mathrm{~mL}$ of a $1 \%$ gold chloride solution was added and left to stir for $3 \mathrm{~min}$. The surface of the nanoparticles was modified with a thiol ligand by first adjusting the $\mathrm{pH}$ of the solution to 7 by the addition of $1.65 \mathrm{~mL}$ of $1 \mathrm{M}$ sodium hydroxide then adding $612 \mu \mathrm{L}$ of a 10 $\mathrm{mM}$ 4-MBA solution in ethanol to a final 4-MBA concentration of $10 \mu \mathrm{M}$. The nanoparticles were allowed to sit for at least 24 $\mathrm{h}$ before use and stored in the dark at $4{ }^{\circ} \mathrm{C}$.

Cluster Formation. The gold nanoparticle clusters were formed using an established method in our group. ${ }^{13,65}$ First, an ultraconcentrated solution of gold nanoparticles was obtained by centrifuging $1 \mathrm{~mL}$ of the gold nanoparticles with $100 \mu \mathrm{L}$ of an immiscible solvent, dichloroethane, in a $2 \mathrm{~mL}$ polypropylene centrifuge tube. The gold nanoparticles pass into the dichloroethane phase during centrifugation, resulting in a small aqueous pellet of ultraconcentrated nanoparticles sitting at the bottom of the centrifuge tube. The aqueous phase is pipetted off to leave the dichloroethane and the ultraconcentrated nanoparticle pellet, which is then sonicated for $10 \mathrm{~s}$. The sonication forms a microemulsion, grouping the nanoparticles into solid spherical clusters. The clusters were pipetted onto glass substrates and allowed to dry. The dried substrates were then soaked for a minimum of $24 \mathrm{~h}$ in a fresh solution of dichloroethene to remove residual contaminants that arose from the contact of dichloroethane with the polypropylene centrifuge tube. After soaking, the substrates were dried under nitrogen and stored for later analysis (see Supplementary Figure 7 for comments on the cluster size distribution).

Grid Patterning of the Glass Substrate. The glass slide was patterned with a grid covering its most central part in order to precisely localize the cluster during the series of consecutive measurements and derive its precise position during the Raman scan. The grid system ( 16 by 16 with a pitch of $10 \mu \mathrm{m}$ ), shown in Supplementary Figure 1a, was fabricated with a direct laser writing lithography technique ${ }^{66}$ followed by the deposition of $40 \mathrm{~nm}$ of indium tin oxide by sputtering (Q150T S sputter system). SEM images were used to confirm the spacing and correct fabrication of the grid and also to determine the sizes of the spherical colloidal superclusters casted on the sample (Supplementary Figures 1 and 3).

Raman Tomography. A confocal Raman microscope (WiTEC) and a bright field objective (Zeiss 100× NA 0.9) were both used to provide the incident linearly polarized illumination and to collect the scattered light from our colloidal spherical cluster. First, Raman scattering spectra were acquired by scanning the laser focus in the $\mathrm{X}-\mathrm{Y}$ plane, and these measurements were repeated at different $\mathrm{Z}$-position across the height of the cluster, allowing us to acquire Raman scattering spectra from the entire structure. The Z-resolution of our measurement was limited by the precision of our piezoelectric motor at $300 \mathrm{~nm}$, while the resolution in the $\mathrm{X}$ and $\mathrm{Y}$ direction was diffraction limited as for an optimized confocal setup (260 $\mathrm{nm}$ for $633 \mathrm{~nm}$ excitation wavelength and $320 \mathrm{~nm}$ for the 785 $\mathrm{nm}$ excitation wavelength). Excitation was provided either by $\mathrm{HeNe}(633 \mathrm{~nm})$ or diode $(785 \mathrm{~nm})$ lasers, with an average power of $0.2 \mathrm{~mW}$ for the $633 \mathrm{~nm}$ excitation laser line and 2.5 $\mathrm{mW}$ for the $785 \mathrm{~nm}$ one.

FDTD Simulations. Due to the high number of gold nanoparticles and the finesse of structural composition, cylindrical geometries were used with Lumerical FDTD software; the electromagnetic properties of spheres can be approximated by cylinders when the polarization is properly considered. ${ }^{13}$ Spectra and near-field maps were calculated for a cylindrical cluster made of gold nanoparticles with a diameter of $40 \mathrm{~nm}$ and spaced by a $2 \mathrm{~nm}$ gap, when excited by an electromagnetic wave coming from the top (same conditions as in the experimental studies). To further prove the robustness of our simulation to map the mode inside supercluster metamaterials, we also simulated clusters containing some defects (see Supplementary Figures 8 and 9).

\section{ASSOCIATED CONTENT}

\section{S Supporting Information}

The Supporting Information is available free of charge on the ACS Publications website at DOI: 10.1021/acsphotonics.7b00504. 
SEM images, FDTD simulations of nonperfectly crystalline clusters, absorption cross section for different cluster diameters, reproducibility of the $3 \mathrm{D}$ tomography measurement (PDF)

\section{AUTHOR INFORMATION}

\section{Corresponding Author}

*E-mail: a.lauri15@imperial.ac.uk.

\section{ORCID $\odot$}

Alberto Lauri: 0000-0001-5517-6511

Emiliano Cortés: 0000-0001-8248-4165

Aliaksandra Rakovich: 0000-0003-1056-5729

\section{Notes}

The authors declare no competing financial interest.

\section{ACKNOWLEDGMENTS}

A.L. thanks the WITec doctoral prize fellowship scheme for financial support. X.X. thanks the Lee Family doctoral prize fellowship scheme for financial support. L.V. would like to acknowledge the support of the H2020-MSCA fellowship. E.C. is supported by a Marie Curie fellowship of the European Commission. V.G. acknowledges ONR Global funding (N62909-15-1-N082). J.B.E. has been funded in part by ERC starting (NanoP) and consolidator (NanoPD) grants along with an EPSRC grant EP/L02098X/1. S.A.M. acknowledges the EPSRC Reactive Plasmonics Programme (EP/MO13812/ 1) and the Lee-Lucas Chair. A.R. would like to acknowledge the Royal Society for their support (UF150542).

\section{REFERENCES}

(1) Grzelczak, M.; Vermant, J.; Furst, E. M.; Liz-Marzán, L. M. Directed Self-Assembly of Nanoparticles. ACS Nano 2010, 4, 35913605.

(2) Whitesides, G. M. Self-Assembly at All Scales. Science (Washington, DC, U. S.) 2002, 295, 2418-2421.

(3) He, J.; Sill, K.; Xiang, H. Self-Directed Self-Assembly of Nanoparticle/Copolymer Mixtures. Nature 2005, 404, 55-59.

(4) Warren, S. C.; Messina, L. C.; Slaughter, L. S.; Kamperman, M.; Zhou, Q.; Gruner, S. M.; DiSalvo, F. J.; Wiesner, U. Ordered Mesoporous Materials from Metal Nanoparticle-Block Copolymer Self-Assembly. Science (Washington, DC, U. S.) 2008, 320, 1748-1752.

(5) Park, J.; Kang, E.; Son, S. U.; Park, H. M.; Lee, M. K.; Kim, J.; Kim, K. W.; Noh, H. J.; Park, J. H.; Bae, C. J.; et al. Monodisperse Nanoparticles of $\mathrm{Ni}$ and $\mathrm{NiO}$ : Synthesis, Characterization, SelfAssembled Superlattices, and Catalytic Applications in the Suzuki Coupling Reaction. Adv. Mater. 2005, 17, 429-434.

(6) Seeman, N. C. Nanomaterials Based on DNA. Annu. Rev. Biochem. 2010, 79, 65-87.

(7) Srivastava, S.; Kotov, N. a. Nanoparticle Assembly for 1D and 2D Ordered Structures. Soft Matter 2009, 5, 1146.

(8) Böker, A.; He, J.; Emrick, T.; Russell, T. P. Self-Assembly of Nanoparticles at Interfaces. Soft Matter 2007, 3, 1231-1248.

(9) Cecchini, M. P.; Turek, V. A.; Demetriadou, A.; Britovsek, G.; Welton, T.; Kornyshev, A. A.; Wilton-Ely, J. D. E. T.; Edel, J. B. Heavy Metal Sensing Using Self-Assembled Nanoparticles at a Liquid-Liquid Interface. Adv. Opt. Mater. 2014, 2 (10), 966-977.

(10) Edel, J. B.; Kornyshev, A. A.; Urbakh, M.; Campus, S. K.; Sw, L.; Kingdom, U.; Aviv, T. Self-Assembly of Nanoparticle Arrays for Use as Mirrors, Sensors, and Antennas. ACS Nano 2013, 7, 9526-9532.

(11) Kuzyk, A.; Schreiber, R.; Fan, Z.; Pardatscher, G.; Roller, E.-M.; Högele, A.; Simmel, F. C.; Govorov, A. O.; Liedl, T. DNA-Based SelfAssembly of Chiral Plasmonic Nanostructures with Tailored Optical Response. Nature 2012, 483, 311-314.

(12) Tian, Y.; Wang, T.; Liu, W.; Xin, H. L.; Li, H.; Ke, Y.; Shih, W. M.; Gang, O. Prescribed Nanoparticle Cluster Architectures and Low-
Dimensional Arrays Built Using Octahedral DNA Origami Frames. Nat. Nanotechnol. 2015, 10, 637-644.

(13) Turek, V. A.; Francescato, Y.; Cadinu, P.; Crick, C. R.; Elliott, L.; Chen, Y.; Urland, V.; Ivanov, A. P.; Hong, M.; Vilar, R.; et al. SelfAssembled Spherical Supercluster Metamaterials from Nanoscale Building Blocks. ACS Photonics 2015, 3, 35.

(14) Zhao, Y.; Shang, L.; Cheng, Y.; Gu, Z. Spherical Colloidal Photonic Crystals. Acc. Chem. Res. 2014, 47, 3632-3642.

(15) Brugarolas, T.; Tu, F.; Lee, D. Directed Assembly of Particles Using Microfluidic Droplets and Bubbles. Soft Matter 2013, 9, 90469058.

(16) Ross, M. B.; Blaber, M. G.; Schatz, G. C. Using Nanoscale and Mesoscale Anisotropy to Engineer the Optical Response of ThreeDimensional Plasmonic Metamaterials. Nat. Commun. 2014, 5, 4090.

(17) Giannini, V.; Fernández-Domínguez, A. I.; Heck, S. C.; Maier, S. a. Plasmonic Nanoantennas: Fundamentals and Their Use in Controlling the Radiative Properties of Nanoemitters. Chem. Rev. 2011, 111, 3888-3912.

(18) Razzari, L.; Toma, A.; Shalaby, M.; Clerici, M.; Zaccaria, R. P.; Liberale, C.; Marras, S.; Al-Naib, I. a. I.; Das, G.; De Angelis, F. Extremely Large Extinction Efficiency and Field Enhancement in Terahertz Resonant Dipole Nanoantennas. Opt. Express 2011, 19, 26088.

(19) Novotny, L. Effective Wavelength Scaling for Optical Antennas. Phys. Rev. Lett. 2007, 98, 266802.

(20) Petersen, C. R.; Møller, U.; Kubat, I.; Zhou, B.; Dupont, S.; Ramsay, J.; Benson, T.; Sujecki, S.; Abdel-Moneim, N.; Tang, Z.; et al. Mid-IR Supercontinuum Covering the $1.4 \mathrm{Mm}$ to $13.3 \mathrm{Mm}$ Molecular Fingerprint Region Using Ultra-High NA Chalcogenide Step-Index Fibre. Nat. Photonics 2014, 8, 1-6.

(21) Steffen, A.; Kogej, T.; Tyrchan, C.; Engkvist, O. Comparison of Molecular Fingerprint Methods on the Basis of Biological Profile Data. J. Chem. Inf. Model. 2009, 49, 338-347.

(22) Mack, D. L.; Cortés, E.; Giannini, V.; Török, P.; Roschuk, T.; Maier, S. A. Decoupling Absorption and Emission Processes in Super Resolution Localization of Emitters in a Plasmonic Hotspot. Nat. Commun. 2017.81451310.1038/ncomms14513

(23) Rang, M.; Jones, A. C.; Fei, Z.; Li, Z. Y.; Wiley, B. J.; Younan, X.; Raschke, M. B. Optical Near-Field Mapping of Plasmonic Nanoprisms. Nano Lett. 2008, 8, 3357-3363.

(24) Koh, A. L.; Bao, K.; Khan, I.; Smith, W. E.; Kothleitner, G.; Nordlander, K. P.; Maier, S. A.; Mccomb, D. W. Electron Energy-Loss Spectroscopy Silver Nanoparticles and Dimers: Influence of Beam Damage and Mapping of Dark Modes. ACS Nano 2009, 3, 30153022.

(25) Liu, N.; Langguth, L.; Weiss, T.; Kästel, J.; Fleischhauer, M.; Pfau, T.; Giessen, H. Plasmonic EIT at the Drude Damping Limit. Conf. Proc. - Lasers Electro-Optics Soc. Annu. Meet 2009, 8, 94-95.

(26) Lobanov, S. V.; Weiss, T.; Dregely, D.; Giessen, H.; Gippius, N. A.; Tikhodeev, S. G. Emission Properties of an Oscillating Point Dipole from a Gold Yagi-Uda Nanoantenna Array. Phys. Rev. B: Condens. Matter Mater. Phys. 2012, 85, 1-6.

(27) Gansel, J. K.; Thiel, M.; Rill, M. S.; Decker, M.; Bade, K.; Saile, V.; von Freymann, G.; Linden, S.; Wegener, M. Gold Helix Photonic Metamaterial as Broadband Circular Polarizer. Science 2009, 325, $1513-1515$.

(28) Ghenuche, P.; Cherukulappurath, S.; Taminiau, T. H.; Van Hulst, N. F.; Quidant, R. Spectroscopic Mode Mapping of Resonant Plasmon Nanoantennas. Phys. Rev. Lett. 2008, 101, 1-4.

(29) Schuck, P. J.; Fromm, D. P.; Sundaramurthy, A.; Kino, G. S.; Moerner, W. E. Improving the Mismatch between Light and Nanoscale Objects with Gold Bowtie Nanoantennas. Phys. Rev. Lett. 2005, 94, 14-17.

(30) Lakowicz, J. R.; Fu, Y. Modification of Single Molecule Fluorescence near Metallic Nanostructures. Laser Photonics Rev. 2009, $3,221-232$

(31) Kühn, S.; Håkanson, U.; Rogobete, L.; Sandoghdar, V. Enhancement of Single-Molecule Fluorescence Using a Gold Nanoparticle as an Optical Nanoantenna. Phys. Rev. Lett. 2006, 97, 1-4. 
(32) Nelayah, J.; Kociak, M.; Stéphan, O.; García de Abajo, F. J.; Tencé, M.; Henrard, L.; Taverna, D.; Pastoriza-Santos, I.; Liz-Marzán, L. M.; Colliex, C. Mapping Surface Plasmons on a Single Metallic Nanoparticle. Nat. Phys. 2007, 3, 348-353.

(33) Vesseur, E. J. R.; De Waele, R.; Kuttge, M.; Polman, A. Direct Observation of Plasmonic Modes in Au Nanowires Using HighResolution Cathodoluminescence Spectroscopy. Nano Lett. 2007, 7, $2843-2846$.

(34) Schnell, M.; García-Etxarri, A.; Huber, a. J.; Crozier, K.; Aizpurua, J.; Hillenbrand, R. Controlling the near-Field Oscillations of Loaded Plasmonic Nanoantennas. Nat. Photonics 2009, 3, 287-291.

(35) Schnell, M.; Garcia-Etxarri, A. Amplitude- and Phase-Resolved Near-Field Mapping of Infrared Antenna Modes by TransmissionMode Scattering-Type Near-Field Microscopy. J. Phys. Chem. C 2010, $114,7341-7345$.

(36) Olmon, R. L.; Krenz, P. M.; Jones, A. C.; Boreman, G. D.; Raschke, M. B. Near-Field Imaging of Optical Antenna Modes in the Mid-Infrared. Opt. Express 2008, 16, 20295.

(37) Lee, S. J.; Guan, Z.; Xu, H.; Moskovits, M. Surface-Enhanced Raman Spectroscopy and Nanogeometry: The Plasmonic Origin of SERS. J. Phys. Chem. C 2007, 111, 17985-17988.

(38) Maier, S. A. Plasmonic Field Enhancement and SERS in the Effective Mode Volume Picture. Opt. Express 2006, 14, 1957-1964.

(39) Nie, S.; Emory, S. R. Probing Single Molecules and Single Nanoparticles by Surface Enhanced Raman Scattering. Science (Washington, DC, U. S.) 1997, 275, 1102-1106.

(40) Adato, R.; Yanik, A. A.; Amsden, J. J.; Kaplan, D. L.; Omenetto, F. G.; Hong, M. K.; Erramilli, S.; Altug, H. Ultra-Sensitive Vibrational Spectroscopy of Protein Monolayers with Plasmonic Nanoantenna Arrays. Proc. Natl. Acad. Sci. U. S. A. 2009, 106, 19227-19232.

(41) Hartstein, A.; Kirtley, J. R.; Tsang, J. C. Enhancement of the Infrared Absorption from Molecular Monolayers with Thin Metal Overlayers. Phys. Rev. Lett. 1980, 45, 201-204.

(42) Quidant, R.; van Hulst, N. F.; Curto, A. G.; Volpe, G.; Taminiau, T. H.; Kreuzer, M. P. Dot Coupled to a Nanoantenna. Science (Washington, DC, U. S.) 2010, 329, 930-933.

(43) Alù, A.; Engheta, N. Hertzian Plasmonic Nanodimer as an Efficient Optical Nanoantenna. Phys. Rev. B: Condens. Matter Mater. Phys. 2008, 78, 1-6.

(44) Novotny, L.; van Hulst, N. Antennas for Light. Nat. Photonics 2011, 5, 83-90.

(45) Mühlschlegel, P.; Eisler, H.-J.; Martin, O. J. F.; Hecht, B.; Pohl, D. W.; Mu, P.; Pohl, D. W. Resonant Optical Antennas. Science (Washington, DC, U. S.) 2005, 308, 1607-1609.

(46) Talley, C. E.; Jackson, J. B.; Oubre, C.; Grady, N. K.; Hollars, C. W.; Lane, S. M.; Huser, T. R.; Nordlander, P.; Halas, N. J. SurfaceEnhanced Raman Scattering from Individual Au Nanoparticles and Nanoparticle Dimer Substrates. Nano Lett. 2005, 5, 1569-1574.

(47) Njoki, P. N.; Lim, I. S.; Mott, D.; Park, H.-Y.; Khan, B.; Mishra, S.; Sujakumar, R.; Luo, J.; Zhong, C.-J. Size Correlation of Optical and Spectroscopic Properties for Gold Nanoparticles. J. Phys. Chem. C 2007, 111, 14664-14669.

(48) Schlücker, S. Surface-Enhanced Raman Spectroscopy: Concepts and Chemical Applications. Angew. Chem., Int. Ed. 2014, 53, 47564795.

(49) Park, H.; Lee, S. B.; Kim, K.; Kim, M. S. Surface-Enhanced Raman Scattering of P-Aminobenzoic Acid at Silver Electrode. J. Phys. Chem. 1990, 94, 7576-7580.

(50) Orendorff, C. J.; Gole, A.; Sau, T. K.; Murphy, C. J. SurfaceEnhanced Raman Spectroscopy of Self-Assembled Monolayers: Sandwich Architecture and Nanoparticle Shape Dependence. Anal. Chem. 2005, 77, 3261-3266.

(51) Królikowska, A.; Kudelski, A.; Michota, A.; Bukowska, J. SERS Studies on the Structure of Thioglycolic Acid Monolayers on Silver and Gold. Surf. Sci. 2003, 532-535, 227-232.

(52) Le Ru, E. C.; Etchegoin, P. G. Rigorous Justification of the $|E| 4$ Enhancement Factor in Surface Enhanced Raman Spectroscopy. Chem. Phys. Lett. 2006, 423, 63-66.
(53) Stockman, M. Nanofocusing of Optical Energy in Tapered Plasmonic Waveguides. Phys. Rev. Lett. 2004, 93, 137404.

(54) Hao, F.; Sonnefraud, Y.; Van Dorpe, P.; Maier, S. A.; Halas, N. J.; Nordlander, P. Symmetry Breaking in Plasmonic Nanocavities: Subradiant LSPR Sensing and a Tunable Fano Resonance. Nano Lett. 2008, 8, 3983-3988.

(55) Lal, S.; Link, S.; Halas, N. J. Nano-Optics from Sensing to Waveguiding. Nat. Photonics 2007, 1, 641-648.

(56) Bohren, C. F.; Huffman, D. M. Absorption and Scattering by a Sphere. Absorpt. Scat. Light Small Part. 1983, 82-129.

(57) Tebbe, M.; Lentz, S.; Guerrini, L.; Fery, A.; Alvarez-Puebla, R. A.; Pazos-Perez, N. Fabrication and Optical Enhancing Properties of Discrete Supercrystals. Nanoscale 2016, 8, 12702-12709.

(58) Camden, J. P.; Dieringer, J. A.; Wang, Y.; Masiello, D. J.; Marks, L. D.; Schatz, G. C.; Van Duyne, R. P. Probing the Structure of SingleMolecule Surface-Enhanced Raman Scattering Hot Spots. J. Am. Chem. Soc. 2008, 130, 12616-12617.

(59) Wang, Y.; Zeiri, O.; Raula, M.; Le Ouay, B.; Stellacci, F.; Weinstock, I. a. Host-Guest Chemistry with Water-Soluble Gold Nanoparticle Supraspheres. Nat. Nanotechnol. 2016, 12, 170-176.

(60) Talley, C. E.; Jusinski, L.; Hollars, C. W.; Lane, S. M.; Huser, T. Intracellular $\mathrm{pH}$ Sensors Based on Surface-Enhanced Raman Scattering. Anal. Chem. 2004, 76, 7064-7068.

(61) Bishnoi, S. W.; Rozell, C. J.; Levin, C. S.; Gheith, M. K.; Johnson, B. R.; Johnson, D. H.; Halas, N. J. All-Optical Nanoscale pH Meter. Nano Lett. 2006, 6, 1687-1692.

(62) Brown, K. R.; Walter, D. G.; Natan, M. J. Seeding of Colloidal $\mathrm{Au}$ Nanoparticle Solutions. 2. Improved Control of Particle Size and Shape. Chem. Mater. 2000, 12, 306-313.

(63) Turkevich, J.; Stevenson, P. C.; Hillier, J. A Study of the Nucleation and Growth Processes in the Synthesis of Colloidal Gold. Discuss. Faraday Soc. 1949, 11, 55.

(64) Frens, G. Controlled Nucleation for the Regulation of the Particle Size in Monodisperse Gold Suspensions. Nature, Phys. Sci. 1973, 241, 20-22.

(65) Turek, V. A.; Elliott, L. N.; Tyler, A. I. I.; Demetriadou, A.; Paget, J.; Cecchini, M. P.; Kucernak, A. R.; Kornyshev, A. A.; Edel, J. B. Self-Assembly and Applications of Ultraconcentrated Nanoparticle Solutions. ACS Nano 2013, 7, 8753-8759.

(66) Braun, A.; Maier, S. A. A Versatile Direct Laser Writing Lithography Technique for Surface Enhanced Infra-Red Spectroscopy Sensors. ACS Sensors 2016, 1, 1155. 